Int. J. Dev. Biol. 58: 743-750 (2014)

doi: 10.1387/ijdb.140296jp

\title{
Comparative analysis of pleurodiran and cryptodiran turtle embryos depicts the molecular ground pattern of the turtle carapacial ridge
}

\author{
JUAN PASCUAL-ANAYA*,1, TATSUYA HIRASAWA', IORI SATO', SHIGEHIRO KURAKU² \\ and SHIGERU KURATANI*,1 \\ ${ }^{1}$ Evolutionary Morphology Laboratory, RIKEN and \\ ${ }^{2}$ Phyloinformatics Unit, RIKEN Center for Life Science Technologies, Kobe, Japan
}

\begin{abstract}
The turtle shell is a wonderful example of a genuine morphological novelty, since it has no counterpart in any other extant vertebrate lineages. The evolutionary origin of the shell is a question that has fascinated evolutionary biologists for over two centuries and it still remains a mystery. One of the turtle innovations associated with the shell is the carapacial ridge (CR), a bulge that appears at both sides of the dorsal lateral trunk of the turtle embryo and that probably controls the formation of the carapace, the dorsal moiety of the shell. Although from the beginning of this century modern genetic techniques have been applied to resolve the evolutionary developmental origin of the CR, the use of different models with, in principle, dissimilar results has hampered the establishment of a common mechanism for the origin of the shell. Although modern turtles are divided into two major groups, Cryptodira (or hidden-necked turtles) and Pleurodira (or side-necked turtles), molecular developmental studies have been carried out mostly using cryptodiran models. In this study, we revisit the past data obtained from cryptodiran turtles in order to reconcile the different results. We also analyze the histological anatomy and the expression pattern of main CR factors in a pleurodiran turtle, the red-bellied short-necked turtle Emydura subglobosa. We suggest that the turtle shell probably originated concomitantly with the co-option of the canonical Wnt signaling pathway into the CR in the last common ancestor of the turtle.
\end{abstract}

KEY WORDS: turtle, evolution, shell, Wnt pathway, Evo-Devo

\section{Introduction}

Turtles are an enigmatic group of animals that have mesmerized zoologists for more than two centuries. Old questions as to the evolutionary origin of turtles and especially the acquisition of the shell still remain to be answered (reviewed by MacCord et al., 2014). The shell is an apomorphy that defines the turtle lineage and it represents a genuine example of morphological novelty sensu stricto (after Müller and Wagner, 1991), since it cannot be obtained by a simple modification of ancestral structures like ribs and the vertebral column. The turtle shell is composed of dorsal and ventral moieties, the so-called carapace and plastron, respectively. Compared with the general tetrapod bauplan, the turtle skeleton is radically different: in the turtle the ribs remain in the dorsal part and grow laterally, instead of ventrally, due to a process that has been called axial arrest (Kuratani et al., 2011). Eventually, unlike in the rest of tetrapods, the rib cage remains open and the shoulder girdle becomes underneath them by folding of the body wall ('Folding Theory'; Kuratani et al., 2011, Nagashima et al., 2009).

The main turtle innovation is the CR, an embryonic structure that appears during early development (although after the phylotypic period characteristic of vertebrate embryos; see Wang et al., 2013) preceding the shell formation. The CR is a bulge of thickened ectoderm overlaying a condensed mesenchyme that first appears in the lateral trunk, dorsal to the lateral somitic frontier running anterior-posteriorly along the flank region, and it has

\footnotetext{
Abbreviations used in this paper: $\mathrm{CNE}$, conserved non-coding element; CR, carapacial ridge.
}

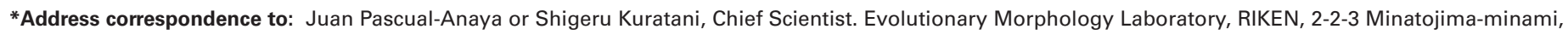
Chuo-ku, Kobe, Hyogo 650-0047, Japan.Tel \& Fax: +81-78-306-3064. E-mail: jpascualanaya@gmail.com; saizo@cdb.riken.jp
}

Supplementary Material (two figures, two tables and one annotations file) for this paper is available at: http://dx.doi.org/10.1387/ijdb.140296jp

Accepted: 19 December 2014.

ISSN: Online 1696-3547, Print 0214-6282 
been proposed to be important for directing the shell development (Burke, 1989, Cebra-Thomas et al., 2005, Nagashima et al., 2007). The CR first appears during stage TK14 (according to the staging table of the Chinese soft-shell turtle, Pelodiscus sinensis -Tokita and Kuratani, 2001-; equivalent to stages Yntema 14 of Chelydra serpentina-Yntema, 1968-; and Greenbaum 15 of the hard-shell red-eared slider Trachemys scripta -Greenbaum, 2002-). The ribs eventually grow into the $\mathrm{CR}$, which in later stages becomes enlarged rostrally and caudally to form a 'ring' that delimits the carapacial margins, and give rise to an open ribcage that will form the scaffold for the carapace. Burke (1989) was the first to notice the similarity between the $\mathrm{CR}$ and the apical ectodermal ridge (AER) of the limbs (thickened ectoderm and condensed underlying mesenchyme, typical of epithelial-mesenchymal interactions).

During the past 14 years, several studies have attempted to describe the genetic bases of the CR (and therefore the carapace) ontogeny with different, and sometimes opposite, results. Loredo et al. (2001) were the first to report a specific expression of developmental genes in the CR. Loredo et al. described the expression of fgf10 in the CR of T. scripta, but the embryos were not staged according to any standardized table, making comparative analyses difficult. Later on, in 2003, Vincent and colleagues found at least one Msx gene expressed in the CR of the European pond turtle, Emys orbicularis, at least from stages $Y 15$ to $Y 19$, not before, and with a decreasing signal in later stages (whether Msx1 or Msx2 is unknown, since the probe used could hybridize with transcripts of both the genes; Vincent et al., 2003). Moreover, while fgf8 was not found in the CR per se (Loredo et al., 2001), it has been described in the distal tip of the ribs as they enter the CR during stage Y14 (Cebra-Thomas et al., 2005). Cebra-Thomas and colleagues also hypothesized that FGF8 from the ribs, and FGF10 from the CR, might form a positive feedback, like that in between the AER and the limb mesenchyme (Rabinowitz and Vokes, 2012). In a recent

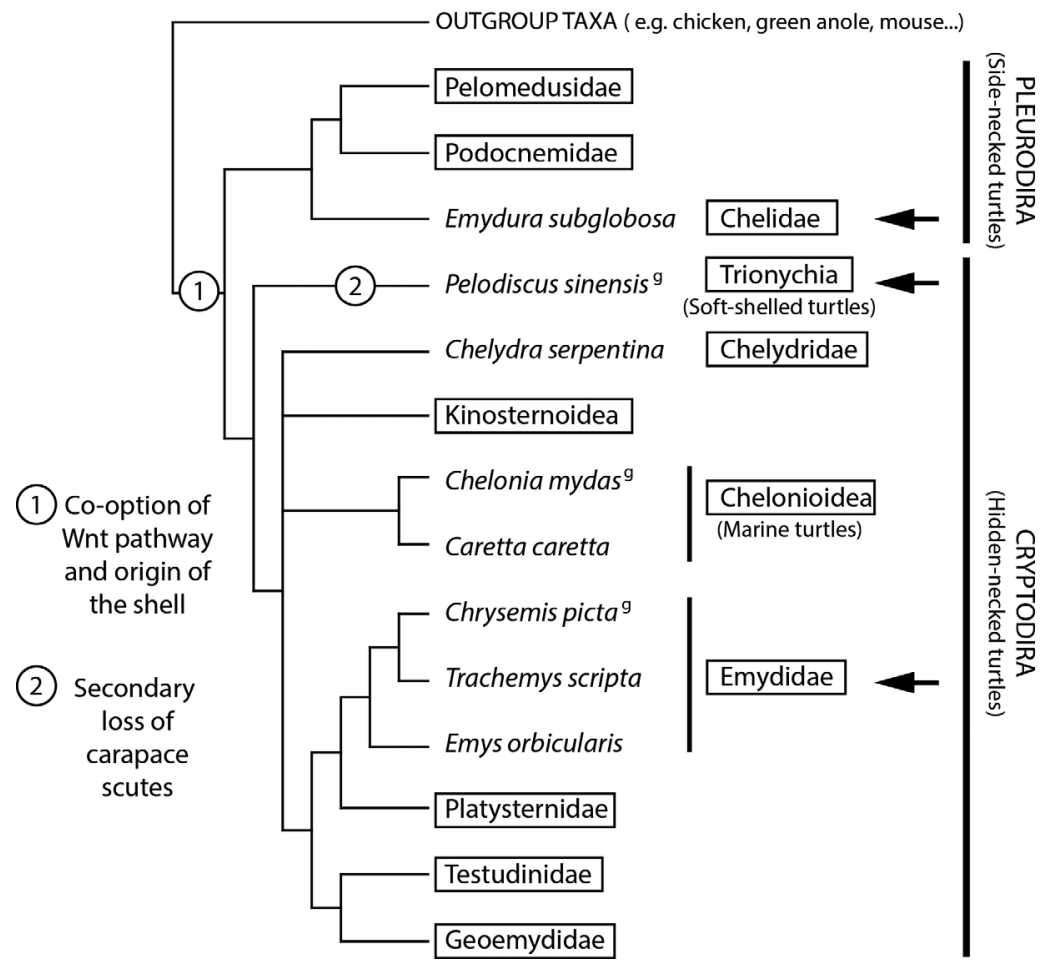

study, Moustakas (2008) found that the gene Gremlin, which form together with BMPs, FGFs and Shh a network in the AER and mesenchyme of the limb bud (Rabinowitz and Vokes, 2012), was expressed in the CR from stage Y14 (Moustakas, 2008). However, this same study concluded that Msx2 (target of the BMP signaling) and $B m p 4$ were not expressed in the $C R$ at stage $Y 14$, but later, and thus play no roles in the CR induction. Moustakas also found that the mesenchymal cells of the CR are derived from the dermomyotomal layer of the somites (Moustakas, 2008). Moustakas and others actually have recently found that Gremlin, Bmp2, Bmp4 and Shh are in fact involved in the formation of the shell epidermal scutes of hard-shelled turtles (Moustakas-Verho et al., 2014).

While these reports described the expression patterns of genes in hard-shell turtles ( T. scriptaand E. orbicularis), the first systematic and comprehensive study of CR-specific molecular markers during its induction was done using the soft-shelled turtle $P$. sinensis (Kuraku et al., 2005). Kuraku and colleagues compared the expression levels of transcripts of the $\mathrm{CR}$ with those of the ventrally adjacent lateral body wall in embryos at stage TK14 and found several genes to be specifically expressed in the CR: Sp5, CRABPI, APCDD1 and Lef-1. Among these genes, Lef-1 is encoding a transcriptions factor that functions as an effector of the canonical Wnt signaling pathway (Behrens et al., 1996). Interestingly, Lef-1 seems to have an important role in controlling the horizontal (not dorsoventral) orientation of the ribs in the carapace (Nagashima et al., 2007). Furthermore, APCDD1 is known to be a target of the canonical Wnt pathway. Accordingly, we found that $\beta$-catenin was specifically translocated into the nucleus of the CR ectodermal cells at stage TK14, when the CR is clearly visible (Kuraku et al., 2005); no equivalent expression patterns have been observed in non-turtle embryos. Although we had previously hypothesized that HGF/c-Met pathway might have had a role in the $\beta$-catenin translocation, $\mathrm{Hgf}$ is, while nearby, actually not expressed in the CR (Kawashima-Ohya et al., 2011, Nagashima et al., 2014). However, the distribution of the HGF protein and a putative role in the $\mathrm{CR}$ formation is unknown, and thus require further investigation. Importantly, upon the analysis of the expression patterns of all Wnt ligands present in the $P$. sinensis genome sequence, we found that Wnt5a is expressed in the CR (Wang et al., 2013), but also in the body wall, precluding its finding in our previous work (Kuraku et al., 2005).

Some of the expression patterns reported in embryos of hard-shelled turtles have not been found in the Chinese soft-shelled turtle. Kuraku and colleagues did not find Msx1, fgf8 or fgf10 expressed in the CR of $P$. sinensis embryos from stages TK13 to 16 (Kuraku et al., 2005). Although some authors have claimed that species-specific

Fig. 1. Simplified phylogeny representing the main groups of modern turtles. The phylogenetic relationships are based on Guillon et al., 2012. Modern turtles are divided into two main groups: Pleurodira, or side-necked turtles, and Cryptodira, or hidden-necked turtles. The soft-shelled turtles form a monophyletic clade, Trionychia. Soft-shell turtles likely lost secondarily the epidermal scutes of the carapace (event 2). The names of those species that have been used in evolutionary developmental, comparative anatomy or genomic studies are indicated. Among them, species with an available genome draft are marked with a ' $g$ '. Arrows indicate the species used in this study. 
differences might account for these differences (for instance, because the models used are either soft-shelled or hard-shelled turtles; see Cebra-Thomas et al., 2005, Gilbert, 2008, Lubick, 2013, Nagashima et al., 2014), we believe that the extraordinary similar developmental pattern of the CR among different turtles must be accounted for by the expression of a common set of genes (Hirasawa et al., 2014). Here, we revisit the past studies reporting expression patterns of developmental genetic markers in the CR of different turtles in order to reconcile the different scenarios into a single developmental origin of the turtle $\mathrm{CR}$, and, by extension, the turtle carapace. We also analyze the expression pattern of major Wnt signaling pathway components in embryos of a side-necked turtle, the red-bellied short-necked turtle E. subglobosa, a representative of the other major group of turtles, Pleurodira, which so far has been excluded from evolutionary molecular developmental studies. Comparisons between pleurodiran and cryptodiran species (the two major extant groups of turtles; Fig. 1) are extremely helpful in inferring the ancestral condition of the turtle body plan and the origin of the carapace. The equivalent expression patterns of Wnt5a, Lef1 and APCDD1 in three turtles ( $P$. sinensis, $T$. scripta and $E$. subglobosa) together with the anatomical study of E. subglobosa sections around $\mathrm{Y} 14$ stage allow us to conclude that the Wnt pathway was acquired in a single event at the origin of modern turtles, and probably was concomitantly co-opted with the origin of the carapace in the last common ancestor of turtles.

\section{Results}

\section{Axial arrest in the side-necked turtle E. subglobosa}

We observed transverse sections of the trunk region of $E$. subglobosa at stage $\mathrm{Y} 14$ (Fig. 2A). At this stage, the CR is distinct, where the overlying ectoderm thickens (Fig. $2 \mathrm{~B}, \mathrm{C}$ ). The secondary body wall component, that is, the dermomyotomal derivatives extend ventrally. The ventral extension of the myotome is distributed in the primary body wall at the level ventral to the CR (Fig. 2 B-D). On the other hand, the rib primordium, identifiable through cell condensation and Alcian-blue staining, is restricted to the levels of the CR and at more dorsal regions (Fig. 2 C,D). This positional relationship of the embryonic structures in E. subglobosa is comparable to those in cryptodiran species that we have previously examined
( $P$. sinensis, T. scripta, Chinemys reevesiiand Caretta caretta) and distinguishable from those in other amniotes (see Hirasawa et al., 2014). Therefore, the axial arrest of the embryonic rib, originally identified in cryptodiran turtles, is also likely to be functioning in the same manner in a pleurodiran species, E. subglobosa.

\section{Fgf8 expression pattern discrepancies revisited}

While Cebra-Thomas et al., (2005) found fgf8 to be expressed in the tip of the ribs around stage $\mathrm{Y} 14$ of $T$. scripta, the same fgf8 expression pattern has not been described in more recent studies by means of whole mounts in P. sinensis (Kuraku et al., 2005) and histological sections in T. scripta(Nagashima et al., 2014) embryos. It is unlikely that they are due to species-specific oddities, because of the extremely similar developmental pattern of turtles at the time of the CR induction (Hirasawa et al., 2014). We have revisited the past turtle fgf8 expression reports in order to reconcile the claimed differences. Surprisingly, transcripts of fgf 8 were detected in $P$. sinensis at stage TK14 in a similar pattern to that found by Gilbert and colleagues (compare Cebra-Thomas et al., 2005, and Fig. 3). Nonetheless, it is remarkable that while turtles have commonly 10 pairs of ribs, the pattern found by Cebra-Thomas et al., shows more than 12 periodic spots along the trunk, and thus fgfo is unlikely expressed in the distal tips of the ribs. Besides, in situ hybridizations on histological sections of $T$. scripta embryos at the level of the ribs showed no expression of fgf8 (Nagashima et al., 2014). Fgf8 is likely expressed in a different and very restricted somitic domain, reminiscent of the normal amniote expression of fgf8 in the somites. This expression might have an important role in the patterning of the ribs, like in other amniotes (Huang et al., 2003). On the other hand, we found this expression pattern in only 1 out of 6 assayed embryos of $P$. sinensis (Fig. 3), indicating a very transient and dynamic expression of fgfo in this region. Also, fgf8 expression level in this putative somitic domain is apparently very low: for instance, the panel shown by Cebra-Thomas and colleagues suggested a strong signal in the mesenchyme of the limbs, although fgf8 is supposedly expressed only in the AER. This was probably due to increased background staining (compare Fig. 3 and Cebra-Thomas et al., 2005). For the abovementioned reasons, the previous works have not shown expression of this gene. We also performed this analysis using embryos of T. scripta

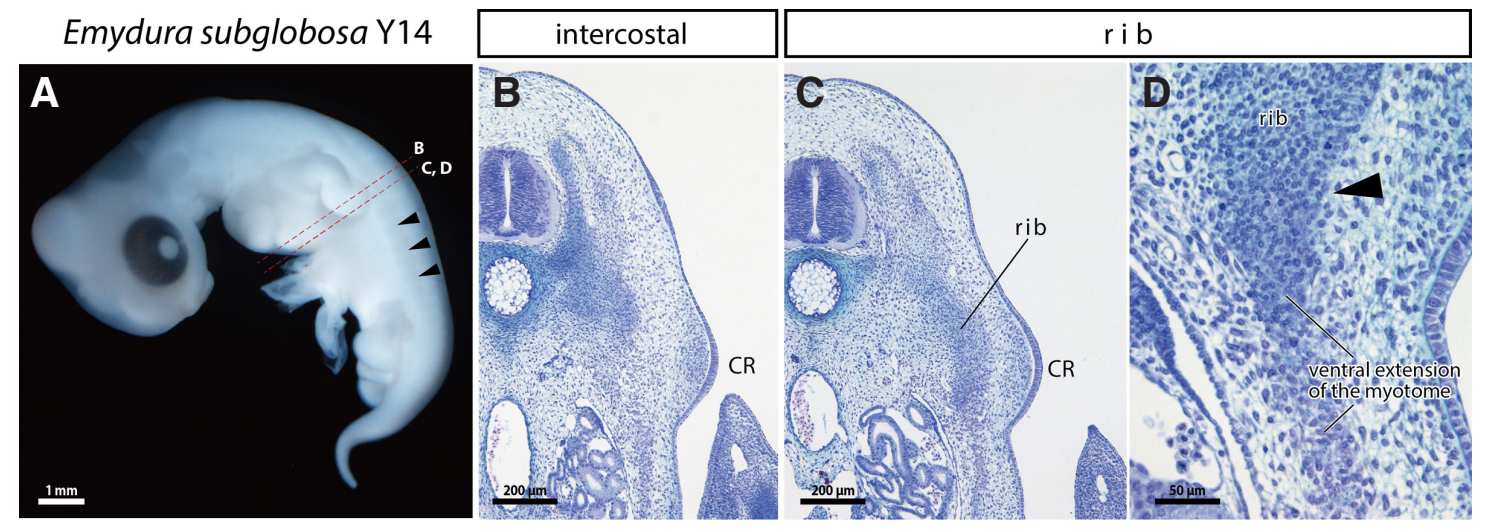

Fig. 2. Axial arrest of the rib primordia at the carapacial ridge (CR) region of the red-bellied short-necked turtle Emydura subglobosa (Pleurodira: Chelidae). (A) Examined specimen (Yntema stage 14) showing the levels of histological (hematoxylin-eosin-Alcian blue staining) sections. Arrowheads indicate the carapacial ridge. (B) Transverse section at an intercostal level. (C) Transverse section at a rib level. (D) Close-up image of the distal end of the rib primordium. Arrowhead indicates the distal end of the rib primordium. 


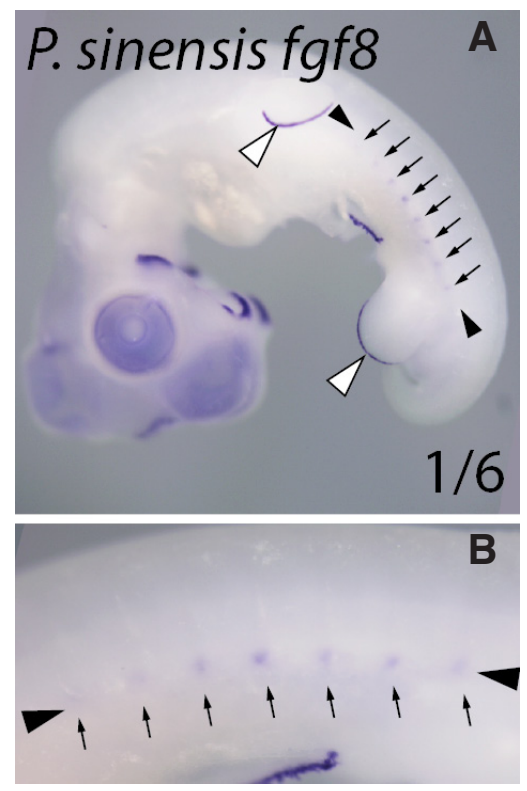

Fig. 3. Expression pattern of fgf8 in Pelodiscus sinensis embryos. (A) Whole mount in situ hybridization of a P. sinensis embryo at stage TK14. 1 out of 6 embryos showed an expression pattern similar to the one previously reported in Trachemys scripta (Vincentet al., 2005). (B) Magnification of the region around the carapacial ridge. Fgf8 is expressed in the nasal placode, maxillary process, AER or both fore-and hindlimbs (white arrowheads) and in a small ventro-posterior compartment of somites (arrows). Arrowheads mark the position of the carapacial ridge.

and E. subglobosa, but because of the lack of embryonic material, and probably the low levels of expression, we were not able to find the above expression pattern, except for general (ancestral) expression domains of fgf8in the posterior somites (Supplementary Fig. S1), as seen in chicken embryos (GEISHAID: FGF8.UAlinear, http://geisha.arizona.edu; Bell et al., 2004, Darnell et al., 2007).

\section{Elements of the canonical Wnt signaling pathway are expressed in the carapacial ridge of all turtles}

Among all the genetic markers reported so far in the $\mathrm{CR}$, one of the most interesting factors is Wnt5a (Wang et al., 2013). Wnt5a is a Wnt ligand, and thus it would occupy, at this moment, the highest position in the hierarchy of the molecular pathway that would possibly control the CR formation. If that is true, Wnt5a is expected to be expressed in the CR from the very beginning of its induction. To test this hypothesis, we analyzed the expression pattern of Wnt5a from stage TK11, corresponding to the phylotypic period for vertebrates (Wang et al., 2013), to stage TK16, when the CR outgrowth is already ongoing (Fig. 4). Remarkably, Wnt5a was not expressed in the region where the $C R$ will form at stages TK11 (Fig. 4 A, A') or TK12 (Fig. 4 B,B'), but was first detected at stage TK13 (Fig. 4 C,C'), just before the CR is formed. Wnt5a continued to be expressed in the CR at stages TK14 (Fig. 4 D,D'), TK15 (Fig. 4 E,E') and TK16 (Fig. 4 F,F'). Therefore, this expression pattern is consistent with a putative role of Wnt5a as a CR inducer.

Next, if Wnt5a, and consequently the Wnt pathway, had a role in the evolutionary origin of the $\mathrm{CR}$, we would expect it to be conserved among different turtles. However, so far the molecular developmental studies have excluded models from one of the two major groups of turtles, Pleurodira (Fig. 1), making the evolutionary scenario of the origin of the CR incomplete. To overcome this lack of information, we obtained several embryos of the side-necked turtle E. subglobosa and performed in situ hybridizations of the genes Wnt5a, Lef-1 and APCDD1 on sections of embryos at stage $Y 14$, and compared with embryos of $T$. scripta, also at stage Y14, and $P$. sinensis at TK14 (Fig. 5). As expected, we detected identical expression patterns of these genes in the CR of all the turtle embryos assayed (Fig. 5), confirming previous reports on cryptodirans (Kuraku et al., 2005, Nagashima et al., 2014, Wang et al., 2013; note that Nagashima and colleagues claimed a slight difference in the case of $A P C D D 1$ that we do not appreciate) and extending it to pleurodirans. At any rate, these results imply that the turtle-specific Wnt5a regulation in the CR as well as axial arrest of ribs would have been present at least in the latest common ancestor of all the turtle-species living today (Fig. 1).

\section{Discussion}

\section{Reconciling differences between hard-shelled and soft-shelled turtles}

Gilbert's and our teams have noted so far assumably speciesspecific differences between embryos of hard-shelled and softshelled turtles (Gilbert, 2008, Kuraku et al., 2005, Nagashima et al., 2014). Previous studies on T. scripta and E. orbicularis have found that $f g f 8$ is expressed in the distal tip of the ribs, and $f g 10$

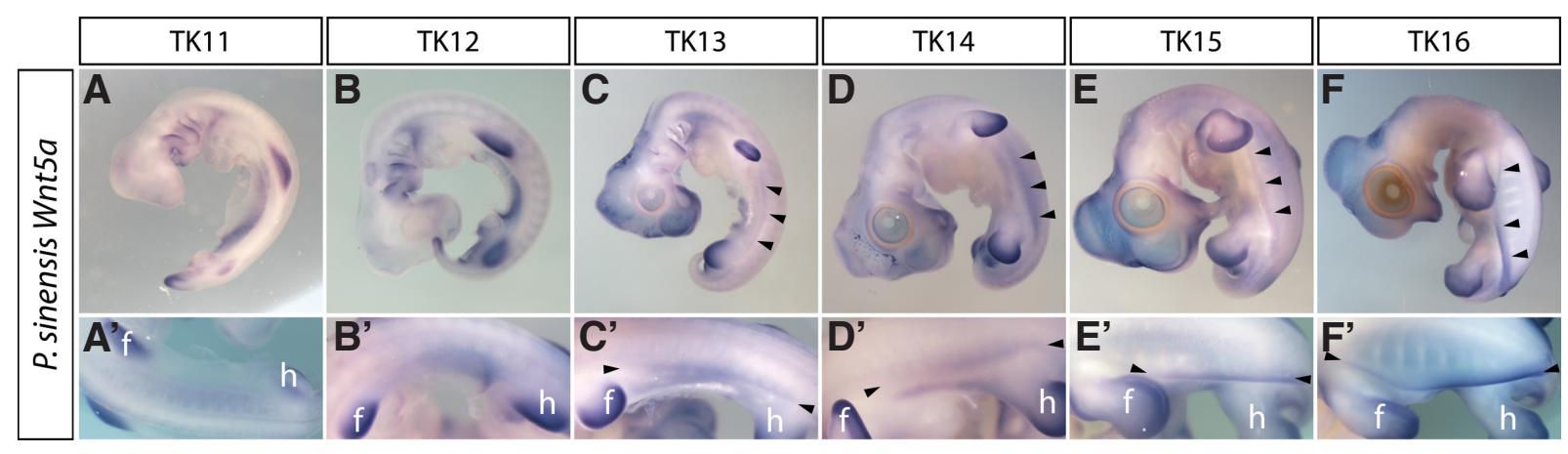

Fig. 4. Expression pattern of Wnt5a during development of Pelodiscus sinensis around the formation of the carapacial ridge (CR) and dorsal shell. Whole mount in situ hybridization of embryos from stage TK11 to TK16 shows that Wnt5a is expressed in the presumptive CR at stage TK13 $\left(\mathbf{C}, \mathbf{C}^{\prime}\right)$ and in the CR through stages TK14 (D, D'), TK15 (E, $\left.\mathbf{E}^{\prime}\right)$ and TK16 (F,F'). It is not expressed in the corresponding region at stages TK11 (A, $\left.\mathbf{A}^{\prime}\right)$ or TK12 (B, $\left.\mathbf{B}^{\prime}\right)$. Arrowheads indicate the position of the CR (presumptive from stage TK13). f, forelimbs; $h$, hindlimbs. Scale bars, $0.5 \mathrm{~mm}$. 


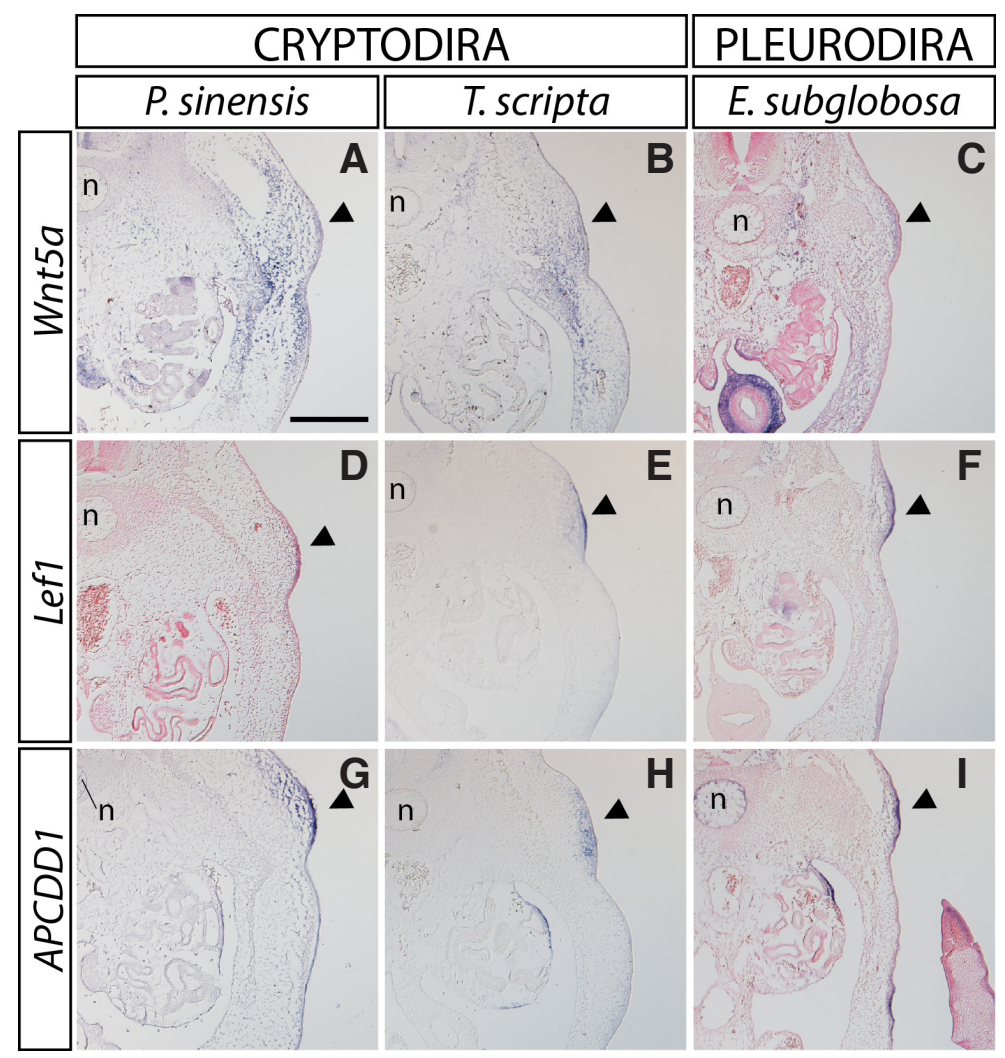

Fig. 5. Expression pattern of Wnt5a, Lef-1 and APCDD1 in representative species of major turtle groups. Panels show histological sections at the level of the interlimb of Pelodiscus sinensis at stage TK14 (A,D,G), Trachemys scripta at stage $Y 14$ (B,E,H) and Emydura subglobosa at stage $Y 14$ (C,F,I). Embryos are sectioned cranio-caudally, so embryonic right is to the left. Dorsal always to the top. $n$, notochord. Scale bar, $200 \mu \mathrm{m}$.

in the CR and limb buds. These results were not found in $P$. sinensis embryos (Kuraku et al., 2005) and were also challenged by a recent study in both $T$. scripta and $P$. sinensis (Nagashima et al., 2014). The first report of fgf10 in the CR used embryos not staged according to a standardized table (like Greenbaum, 2002, or Yntema, 1968), but reported the expression in 23-day and 29-day old embryos (Loredo et al., 2001; note that data for the 23-day old embryo was not shown). However, according to the histological anatomy of the sections presented by Loredo and colleagues (29-day old embryo), and the timetable described by Greenbaum (2002), where the time of incubation to reach a given stage is variable, it seems that this study was conducted in embryos much older than Y14, significantly after the appearance of CR. This would explain why other reports have not been able to find fgf10 in the CR of embryos at Y14, suggesting that it has no role in the induction of the CR per se. Regarding fgf8 expression, as we have shown here, the expression pattern found by Cebra-Thomas and colleagues (Cebra-Thomas et al., 2005) likely corresponds to the ancestral expression of fgf8 in the somites, and not the ribs. The level of fgf8 expression in this somitic domain is probably very low and temporarily short, explaining why have not been found in other studies (Kuraku et al., 2005, Nagashima et al., 2014).

Last, a third reported discrepancy is the expression of Msx genes. Vincent et al. (2003) found that at least one Msx gene was expressed in the CR of T. scripta from stages Y15 to Y19. Kuraku et al. claimed that $P$. sinensis CR did not express Msx1 and Msx2 genes (Kuraku et al., 2005). This difference would be explained, first, by the fact that Vincent et al. (2003) used a very short riboprobe based on the homeobox region of the gene, with a high identity between Msx1 and Msx2, and thus would have easily cross-hybridized with both Msx 1 and Msx2 (as assayed by Southern blot in Vincent et al., 2003). Second, Kuraku et al. (2005) found that the exact sequence from Vincent and others' study (' $E-M s x^{\prime}$ ) corresponded to Msx1, and thus comprehensively study the expression of $P$. sinensis Msx1 from stages TK13 to TK16. They also analyzed $P$. sinensis Msx2 expression, but at stage TK13. Later, Moustakas (2008) concluded that Msx2, the gene actually expressed in the $\mathrm{CR}$, is not involved in the CR induction because it was expressed in the CR later in development, explaining why it was also not found by Kuraku et al. (2005) in earlier stages. In fact, a recent report by Moustakas-Verho and colleagues showed that Msx2 might have a key role in the formation of epidermal scutes in hard-shelled turtles, a developmental module that seems arrested in soft-shelled turtles (Moustakas-Verho et al., 2014). Therefore, we conclude that there is no discrepancy in Msx data between hard-shelled and soft-shelled turtles.

As we have described here, the reported dissimilarities between hard- and soft-shelled turtles are most likely due to: differences in the stages assayed ( $f g f 10)$; genes involved in different developmental modules (scaffold of the carapace or epidermal scutes, like Msx2); non-specific probes (Msx probe based on homeobox); and apparently very dynamic spatiotemporal expressions as we have seen in the case of fgfo. Care should be taken in the future as to the expression patterns found and the conclusions to be drawn from them. We propose that future research must be very specific in (i) the stage of embryos assayed, always according to standardized tables that facilitates the cross-species comparisons; (ii) utilization of unequivocal probes, with special care in the case of close paralogues (for instance, by avoiding probes based in conserved domains); and (iii), when possible, use embryos from different turtle clades, in order to be able to, on the one hand, infer common patterns, and on the other to distinguish between different developmental modules, as soft-shelled turtles are expected to have lost patterns seen related with scutes development (see Moustakas-Verho et al., 2014).

\section{Wnt pathway and the evolutionary origin of the carapacial ridge}

Common developmental patterns generally denote a common evolutionary origin. As we have shown here, embryos of a pleurodiran turtle have the rib development axially arrested, with a conserved topographical relationship of different anatomical elements in the same position as other turtles (Hirasawa et al., 2014). This suggests that the development of the shell by the axial arrest of the rib cage and the folding of the body wall is the mechanism by which the carapace was formed in the common ancestors of turtles (Hirasawa et al., 2014, Kuratani et al., 2011, Nagashima et al., 2009). The turtle last common ancestor would probably have had epidermal scutes over the endoskeleton scaffold of the carapace, but the developmental module underlying the formation of the scutes comes later than the CR induction in development 
(Moustakas-Verho et al., 2014), and whether it has been indeed conserved between cryptodiran and pleurodiran still needs further research. On the other hand, we have been able to reproduce our previous reports of Wnt pathway elements in the CR in two hard-shell turtles: the cryptodiran T. scripta and the pleurodiran $E$. subglobosa. The conservation of these turtle-specific expression domains during the $\mathrm{CR}$ induction between turtles from the two main branches of the turtle phylogeny (Fig. 4; Kuraku et al., 2005) allows us to infer that it originated before the last common ancestor of modern turtles, suggesting the Wnt pathway as the main candidate signaling system involved in the evolutionary origin of the carapace. However, empirical evidence about a direct relationship between the Wnt pathway and the CR development remains to be found. Functional analyses will clarify the role of Wnt5a in the CR induction; for instance, by ectopically applying Wnt5a in the lateral trunk of the chicken embryo, or by application of Wnt inhibitors in the turtle embryo. Nonetheless, even negative results using this transphyletic strategy, like the ectopic expression of other factors, are not easily interpretable. Wnt5a is a known non-canonical $\beta$-catenin-independent ligand, and thus one would not expect Wnt5a to activate the canonical pathway in the CR (Kuraku et al., 2005). Importantly, the function of a Wnt ligand does not directly depends upon the ligand per se, but on the receptor repertoire present in the cell which the signaling is directed to, and accordingly, Wnt5a has been reported to activate the canonical Wnt pathway depending on the receptor context (Mikels and Nusse, 2006). Therefore, just expressing the ligand in the correspondent area might not be enough to induce an ectopic $\mathrm{CR}$, but the corresponding receptors are also needed.

\section{Regulatory genomics of the turtle shell}

One of the main unresolved questions in evolutionary developmental biology is the mechanistic scenario behind the morphological novelties. Since the discovery of the conserved 'toolkit' genes, changes on the regulation of these genes, that allow the co-option and shuffling of developmental modules into new areas, have been proposed as the main evolutionary cause of evolutionary innovations (Carroll et al., 2001). However, the exact mechanisms by which these changes in the regulatory regions can lead to morphological novelties remain a mystery.

The turtle shell is an extraordinarily excel-

Fig. 6. Phylogenetic footprinting of the genomic region surrounding Wnt5a. VISTA plots of AVID alignments of the genomic sequences encompassing the areas between the most proximate upstream gene (ERC2) and the closest downstream gene (CACNA2D3) of ten gnathostome vertebrates. From top to bottom: Pelodiscus sinensis (base genome), Chelonia mydas, Chrysemys picta bellii, Gallus gallus, Taenopygia guttata, Anolis carolinensis, Homo sapiens, Mus musculus, Monodelphis domestica, Danio rerio. On the top, the genomic region of $\mathrm{P}$. sinensis is represented by a black line, with coding genes and their transcriptional orientation illustrated by greyarrows. Turtle-specific conserved non-coding sequences are outlined by a red rectangle. Turtle silhouettes in black; other birds and reptiles in red; mammals in blue; zebrafish in green. Peaks are color-coded only when the identity percentage is equal to or higher than $70 \%$ over a $100 \mathrm{bp}$ window with respect to the $P$. sinensis sequence. Purple peaks represent exons; turquoise, UTRs; and pink, non-coding sequences. 
lent model to study the linkage between the changes in the genomic landscape and the morphological output that they produce. We have shown here that the Wnt pathway was likely co-opted into the CR before the last common ancestor of modern turtles. But, which exact genomic changes can account for this co-option? If the co-option of the Wnt pathway into new territories in the turtle was accompanied by changes in the regulatory regions of the genes involved, then, we would expect these regulatory sequences to be present only in turtles, and not in other vertebrates. With this assumption in mind, turtle-specific conserved non-coding elements (CNEs), detected for example by comparative genomics analyses, would be strong candidates for such regulatory regions (see review by GomezSkarmeta et al., 2006). Recently, the genome draft sequences of three different turtles have been reported (Shaffer et al., 2013, Wang et al., 2013). Multiple sequence alignments and phylogenetic footprinting analyses between these three turtles and other vertebrates' genomic sequences would allow us to identify these CNEs. As an example of the potential of this kind of analyses, we have aligned and visualized the genomic regions surrounding the Wnt5a gene from 10 different vertebrates by means of VISTA plot analysis (Fig. 6 and Suppl. Fig. S2). We found 354 turtle-specific CNEs (see Methods), of which $28 \%$ are within the upstream region of Wnt5a (between ERC2 and Wnt5a) and, interestingly, one of them is placed within the Wnt5aintron (Suppl. Fig. S2). Deciphering whether these CNEs are indeed regulatory elements or not requires further experiments, like for example reporter assays in the turtle embryo. One would expect that some of these putative regulatory sequences, or combination of them, are able to recapitulate the expression of Wnt5a into the corresponding area of other amniote embryos. It is tempting to think that if we are able to identify the significant set of the regulatory regions of those genes with key roles in the CR, we would then be able to phenocopy a CR in a non-turtle amniote embryo where it is absent.

\section{Materials and Methods}

\section{Animal care and sample collection}

$P$. sinensis eggs were purchased form a local farm in Japan. Eggs were cleaned upon arrival to the laboratory, and cultured at 30 degrees. The embryos staged according to Tokita and Kuratani (2001). Eggs of $T$. scripta were obtained from the Suma Aquarium, Kobe, Japan. The eggs were cleaned and cultured at 28 degrees. $T$. scripta embryos were staged according to Yntema, 1968 or Greenbaum, 2002. Embryos of E. subglobosa, were gifted by $\mathrm{H}$. Shibata.

Experimental procedures and animal care were conducted according to guidelines approved by the RIKEN Animal Experiments Committee (Approval ID H14-23-20).

\section{Histological analysis}

An embryo of $E$. subglobosa was fixed with Serra's fixative, substituted with graded methanol series, and embedded in paraffin. Serial histological sections $(6 \mu \mathrm{m})$ of the embryo were prepared, and stained with Alcian-blue, hematoxylin and eosin.

\section{Cloning and in situ hybridizations}

Total RNA from $P$. sinensis embryos at different stages from neurula to stage TK23, from one embryo of T. scripta at stage Y17 and from the brain of a single juvenile of $E$. subglobosa were extracted using the RNeasy Mini or Midi Kit (QIAGEN). cDNA was then synthesized using the SuperScript ${ }^{\circledR}$ III First-Strand Synthesis System (Invitrogen; Life Technologies) with an anchored oligo (d) $\mathrm{T}_{20}$ primer. Primers for $P$. sinensis genes were designed based on the gene sequences from Ensembl Chinese Soft-Shelled Turtle
Genome Browser (http://www.ensembl.org/Pelodiscus_sinensis) or previous reports (Kuraku et al., 2005) (see Supplementary Table S1). For the sake of reproducibility, the $P$. sinensis Wnt5a probe used in this study was different from the one previously reported by our lab (Wang et al., 2013). T. scripta APCDD1, Wnt5a, Lef-1 and fgf8 orthologues were obtained by tBLASTn using the corresponding $P$. sinensis proteins as queries against the available T. scriptatranscriptome (Kaplinsky et al., 2013). Corresponding accession numbers of the $T$. sripta genes obtained from the transcriptome are available in the Suplementary Table S1. A cDNA fragment for the $E$. subglobosa APCDD1 gene was amplified with 3' rapid amplification of cDNAends (3'RACE) System (Invitrogen; Life Technologies) employing two forward degenerate primers. The first PCR was performed with the forward primer 5'-GAG GAG CTT TWY YTN GGN GAY ATH CA-3' and the abridged universal amplification primer (AUAP) supplied in the 3'RACE System kit, which was followed by a nested PCR employing the forward primer 5'-CTT TAC CTN GGN GAY ATH CAY ACN GA-3' together with the AUAP. Next, non-degenerate specific primers were designed to clone a smaller fragment that was used for in situ hybridization (see Supplementary Table S1). Non-degenerate primers for E. subglobosa Lef-1, Wnt5a and fgf8 genes were designed based on high-identity regions of DNA sequence alignments between T. scripta, C. picta, Chelonia mydas, P. sinensis and chicken ( $G a l-$ Ius gallus) counterparts. E. subglobosa gene sequences were submitted to the NCBI GenBank database under the accession numbers KP114658 $-\mathrm{KP} 114661$. The fragments obtained in all cases were subcloned into the $\mathrm{pCRII/TOPO} \mathrm{vector} \mathrm{of} \mathrm{the} \mathrm{Dual} \mathrm{Promoter} \mathrm{TA-cloning} \mathrm{System} \mathrm{(Invitrogen;} \mathrm{Life}$ Technologies), sequenced from both extremes of the TA-cloning site with universal primers M13F and M13R, and sequences were blasted against the GenBank database to check the orthology. All primers used in this study are available in the Supplementary Table 1. DIG-labeled riboprobes were synthesized using the DIG labeling kit (Roche) according to the manufacturer specifications. Sense probes are not good negative controls due to the possibility of antisense expression, thus the specific pattern found with all probes served as controls for each other. For instance, the lack of expression of fgf8 in the $\mathrm{CR}$ is a negative control for the expression in the CR of Wnt5a, Lef1 and APCDD1 and vice versa. Some of them have also been published previously, suggesting that these results are reproducible.

Embryos for whole mount and section in situ hybridizations were fixed with $4 \%$ paraformaldehyde in phosphate-buffered saline (PBS) at 4 degrees, 16-24 hours. Subsequently, embryos were dehydrated in serial increasing concentrations of methanol and store at -30 degress. Whole mount and section in situ hybridizations were performed as previously described (Kuraku et al., 2005).

\section{Phylogenetic footprinting}

Genomic sequences from the closest upstream to downstream genes of Wnt5a were downloaded from Ensembl (http://www.ensembl.org), Ensembl Pre! (http://pre.ensembl.org, for $C$. picta) or NCBI (for $C$. mydas), together with the corresponding VISTAannotation, from 10 different vertebrates: three turtles (P. sinensis, C. picta, and C. mydas), two birds (G. galus, Taeniopygia guttata), a lizard (Anolis carolinensis), three mammals (Homo sapiens, Mus musculus, Monodelphis domestica) and a fish (Danio rerio). In case that a gene was not annotated, we identified it by means of tBLASTn using the closest species' protein sequence as query, and the gene model and its exon coordinates were predicted using Exonerate v.2.2.0 with options "--showtargetgff --model protein2genome" using the same query protein sequence as reference (Slater and Birney, 2005). Annotation was manually done for the whole locus in the case of $C$. mydas. Repetitive elements were masked using RepeatMasker v.4.0.3 (Smit et al., 1996-2010) using the Repbase Update 20130422 (Jurka et al., 2005). The genomic sequences were aligned by means of AVID with default parameters (Bray et al., 2003) and conservation plots generated by the command-line version of VISTA v1.4.26 (Frazer et al., 2004), defining conserved elements as those with at least a $70 \%$ identity over a $100 \mathrm{bp}$-window. The coordinates of the CNEs found were formatted to GFF format using an in-house Perl script (available in https://github.com/JPascualAnaya/VISTA2GFF). Only intersections 
of CNEs present in both $P$. sinensis - $C$. mydas and $P$. sinensis $-C$. picta comparisons, if they overlapped at least $90 \%$ of their length, were further considered. The resulted regions were merged if they were contiguous or overlapped at least 1 nucleotide. Conserved regions from either turtle comparison $(P$. sinensis $-C$. mydas or $P$. sinensis $-C$. picta) with any other vertebrate but not present in the three turtles were discarded. Finally, the resulted conserved regions were merged if separated by less than $101 \mathrm{bp}$. Intersections, overlapping and merging of the conserved regions between different datasets were assayed by bedtools v2.17 (Quinlan and Hall, 2010). The corresponding genomedraft versions, chromosomes or scaffolds IDs and sequence coordinates used are available in the Supplementary Table S2. All gene coordinates in VISTA format are available in Supplementary File S1.

\section{Acknowledgments}

The authors are indebted to Dr. Naoki Kamezaki, Mari Taniguchi and Kanako Mine from the SumaAquarium (Kobe, Japan) for providing T. scripta embryos, and to Hiroki Shibata, Eiji Nitasaka and Hiroshi Nagashima for providing E. subglobosa embryos. This work has been funded by a KAKENHI Grant-in-aid for Scientific Research on Innovative Areas to S. Kuratani and a Grant-in-Aid for Young Scientists B to JP-A of the Japanese Society for the Promotion of Science and the Ministry for Education, Culture, Sports, Science and Technology (Japan).

\section{References}

BEHRENS, J., VON KRIES, J.P., KUHL, M., BRUHN, L., WEDLICH, D., GROSSCHEDL, R. and BIRCHMEIER, W. (1996). Functional interaction of beta-catenin with the transcription factor LEF-1. Nature 382: 638-642.

BELL, G.W., YATSKIEVYCH, T.A. and ANTIN, P.B. (2004). GEISHA, a whole-mount in situhybridization gene expression screen in chicken embryos. Dev Dyn229:677-687.

BRAY, N., DUBCHAK, I. and PACHTER, L. (2003). AVID: A global alignment program. Genome Res 13: 97-102.

BURKE, A. (1989). Development of the turtle carapace: implications for the evolution of a novel bauplan. J Morphol 199: 363-378.

CARROLL, S.B., GRENIER, J.K. and WEATHERBEE, S.D. (2001). From DNA to Diversity: Molecular Genetics and the Evolution of Animal Design. Blackwell Science.

CEBRA-THOMAS, J., TAN, F., SISTLA, S., ESTES, E., BENDER, G., KIM, C., RICCIO, P. and GILBERT, S.F. (2005). How the turtle forms its shell: a paracrine hypothesis of carapace formation. J Exp Zool B Mol Dev Evol 304: 558-569.

DARNELL, D.K., KAUR, S., STANISLAW, S., DAVEY, S., KONIECZKA, J.H., YATSKIEVYCH, T.A. and ANTIN, P.B. (2007). GEISHA: an in situ hybridization gene expression resource for the chicken embryo. Cytogenet Genome Res 117: 30-35.

FRAZER, K.A., PACHTER, L., POLIAKOV, A., RUBIN, E.M. and DUBCHAK, I. (2004). VISTA: computational tools for comparative genomics. Nucleic Acids Res 32: W273-W279.

GILBERT, S.F., CEBRA-THOMAS, J. A., BURKE, A. C. (2008). How the turtles gets its shell. In Biology of Turtles, (ed. JEANETTE WYNEKEN, M. H. G., VINCENT BELS). CRC Press, Boca Raton, pp.1-19.

GOMEZ-SKARMETA, J.L., LENHARD, B. and BECKER, T.S. (2006). New technologies, new findings, and new concepts in the study of vertebrate cis-regulatory sequences. Dev Dyn 235: 870-885.

GREENBAUM, E. (2002). A standardized series of embryonic stages for the emydid turtle Trachemys scripta. Can J Zool 80: 1350-1370.

HIRASAWA, T., PASCUAL-ANAYA, J., KAMEZAKI, N., TANIGUCHI, M., MINE, K. and KURATANI, S. (2015). The evolutionary origin of the turtle shell and its dependence on the axial arrest of the embryonic rib cage. J Exp Zool B Mol Dev Evol. 324: 194-207.

HUANG, R., STOLTE, D., KURZ, H., EHEHALT, F., CANN, G.M., STOCKDALE, F.E., PATEL, K. and CHRIST, B. (2003). Ventral axial organs regulate expression of myotomal Fgf-8 that influences rib development. Dev Biol 255: 30-47.

JURKA, J., KAPITONOV, V.V., PAVLICEK, A., KLONOWSKI, P., KOHANY, O. and WALICHIEWICZ, J. (2005). Repbase Update, a database of eukaryotic repetitive elements. Cytogenet Genome Res 110: 462-7.
KAPLINSKY, N.J., GILBERT, S.F., CEBRA-THOMAS, J., LILLEVALI, K., SAARE, M., CHANG, E.Y., EDELMAN, H.E., FRICK, M.A., GUAN, Y., HAMMOND, R.M. et al., (2013). The Embryonic Transcriptome of the Red-Eared Slider Turtle (Trachemys scripta). PLoS One 8: e66357.

KAWASHIMA-OHYA, Y., NARITA, Y., NAGASHIMA, H., USUDA, R. and KURATANI, S. (2011). Hepatocyte growth factor is crucial for development of the carapace in turtles. Evol Dev 13: 260-268.

KURAKU, S., USUDA, R. and KURATANI, S. (2005). Comprehensive survey of carapacial ridge-specific genes in turtle implies co-option of some regulatory genes in carapace evolution. Evol Dev 7: 3-17.

KURATANI, S., KURAKU, S. and NAGASHIMA, H. (2011). Evolutionary developmental perspective for the origin of turtles: the folding theory for the shell based on the developmental nature of the carapacial ridge. Evol Dev 13: 1-14.

LOREDO, G.A., BRUKMAN, A., HARRIS, M.P., KAGLE, D., LECLAIR, E.E., GUTMAN, R., DENNEY, E., HENKELMAN, E., MURRAY, B.P., FALLON, J.F. et al., (2001). Development of an evolutionarily novel structure: fibroblast growth factor expression in the carapacial ridge of turtle embryos. J Exp Zool 291: 274-281.

LUBICK, N. (2013). Development. Biologists tell dueling stories of how turtles get their shells. Science 341: 329 .

MACCORD, K., CANIGLIA, G., MOUSTAKAS-VERHO, J.E. and BURKE, A.C. (2014). The dawn of chelonian research: Turtles between comparative anatomy and embryology in the 19th century. J Exp Zool B Mol Dev Evol. 324: 169-180.

MIKELS, A.J. and NUSSE, R. (2006). Purified Wnt5a protein activates or inhibits beta-catenin-TCF signaling depending on receptor context. PLoS Biol 4: e115.

MOUSTAKAS, J.E. (2008). Development of the carapacial ridge: implications for the evolution of genetic networks in turtle shell development. Evol Dev 10: 29-36.

MOUSTAKAS-VERHO, J.E., ZIMM, R., CEBRA-THOMAS, J., LEMPIAINEN, N.K., KALLONEN, A., MITCHELL, K.L., HAMALAINEN, K., SALAZAR-CIUDAD, I., JERNVALL, J. and GILBERT, S.F. (2014). The origin and loss of periodic patterning in the turtle shell. Development 141: 3033-3039.

MÜLLER, G. and WAGNER, G. (1991). Novelty in Evolution: Restructuring the Concept. Ann. Rev. Ecol. Systemat. 22: 229-256.

NAGASHIMA, H., KURAKU, S., UCHIDA, K., OHYA, Y.K., NARITA, Y. and KURATANI, S. (2007). On the carapacial ridge in turtle embryos: its developmental origin, function and the chelonian body plan. Development 134: 2219-2226.

NAGASHIMA, H., SHIBATA, M., TANIGUCHI, M., UENO, S., KAMEZAKI, N. and SATO, N. (2014). Comparative study of the shell development of hard- and soft-shelled turtles. J Anat 225: 60-70.

NAGASHIMA, H., SUGAHARA, F., TAKECHI, M., ERICSSON, R., KAWASHIMAOHYA, Y., NARITA, Y. and KURATANI, S. (2009). Evolution of the turtle body plan by the folding and creation of new muscle connections. Science 325: 193-196.

QUINLAN, A.R. and HALL, I.M. (2010). BEDTools: a flexible suite of utilities for comparing genomic features. Bioinformatics 26: 841-842.

RABINOWITZ,A.H. and VOKES, S.A. (2012). Integration of the transcriptional networks regulating limb morphogenesis. Dev Biol 368: 165-180.

SHAFFER, H.B., MINX, P., WARREN, D.E., SHEDLOCK, A.M., THOMSON, R.C., VALENZUELA, N., ABRAMYAN, J., AMEMIYA, C.T., BADENHORST, D., BIGGAR, K.K. et al., (2013). The western painted turtle genome, a model for the evolution of extreme physiological adaptations in a slowly evolving lineage. Genome Biol14:R28.

SLATER, G.S. and BIRNEY, E. (2005). Automated generation of heuristics for biological sequence comparison. BMC Bioinformatics 6: 31 .

SMIT, A.F.A., HUBLEY, R. and GREEN, P. (1996-2010). RepeatMasker Open-3.0., $<$ http://www.repeatmasker.org>

TOKITA, M. and KURATANI, S. (2001). Normal embryonic stages of the Chinese softshelled turtle Pelodiscus sinensis (Trionychidae). Zoological Science 18: 705-716.

VINCENT, C., BONTOUX, M., LEDOUARIN, N.M., PIEAU, C. and MONSORO-BURQ, A.H. (2003). Msx genes are expressed in the carapacial ridge of turtle shell: a study of the European pond turtle, Emys orbicularis. Dev Genes Evol 213: 464-469.

WANG, Z., PASCUAL-ANAYA, J., ZADISSA, A., LI, W., NIIMURA, Y., HUANG, Z., LI, C., WHITE, S., XIONG, Z., FANG, D. et al., (2013). The draft genomes of soft-shell turtle and green sea turtle yield insights into the development and evolution of the turtle-specific body plan. Nat Genet 45: 701-706.

YNTEMA, C.L. (1968). A series of stages in the embryonic development of Chelydra serpentina. J Morphol 125: 219-251. 


\section{Further Related Reading, published previously in the Int. J. Dev. Biol.}

The morphogenesis of evolutionary developmental biology

Scott F Gilbert

Int. J. Dev. Biol. (2003) 47: 467-477

Transcriptional regulation and the evolution of development

Gregory A Wray

Int. J. Dev. Biol. (2003) 47: 675-684

Reptile scale paradigm: Evo-Devo, pattern formation and regeneration

Cheng Chang, Ping Wu, Ruth E. Baker, Philip K. Maini, Lorenzo Alibardi and Cheng-Ming Chuong

Int. J. Dev. Biol. (2009) 53: 813-826

Evo-Devo of amniote integuments and appendages

Ping Wu, Lianhai Hou, Maksim Plikus, Michael Hughes, Jeffrey Scehnet, Sanong Suksaweang, Randall Widelitz, Ting-Xin Jiang and Cheng-Ming Chuong

Int. J. Dev. Biol. (2004) 48: 249-270

The place of phylogeny and cladistics in Evo-Devo research Maximilian J Telford and Graham E Budd

Int. J. Dev. Biol. (2003) 47: 479-490

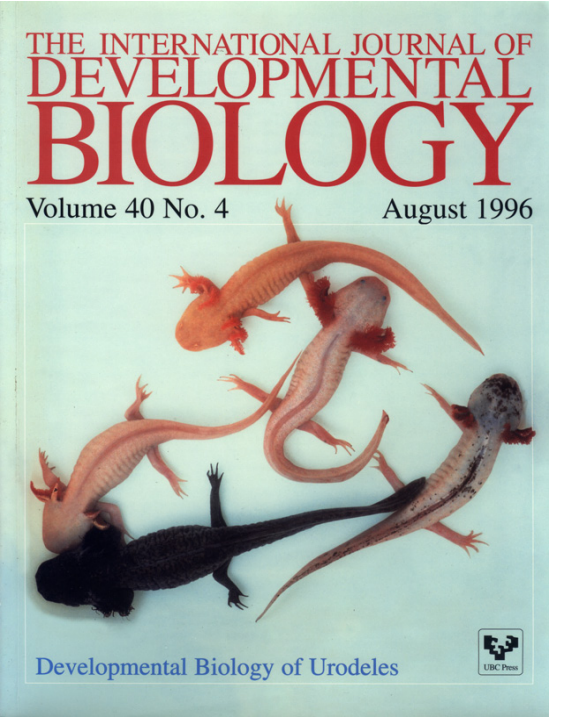

5 yr ISI Impact Factor $(2013)=2.879$
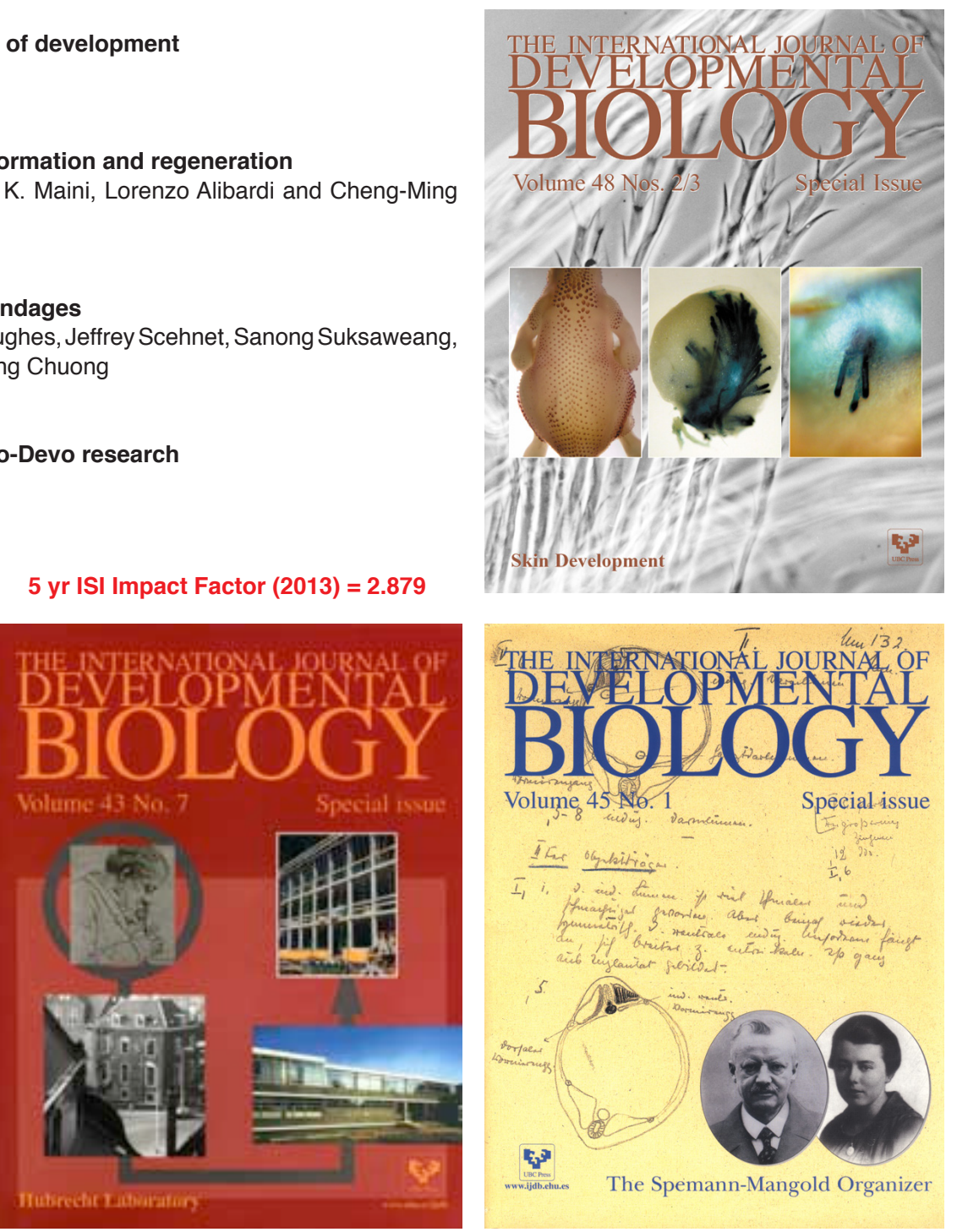